\title{
Hábitos alimenticios del pez Lagodon rhomboides (Perciformes: Sparidae) en la laguna costera de Chelem, Yucatán, México
}

\author{
Walter Gabriel Canto-Maza \& María Eugenia Vega-Cendejas \\ Laboratorio de Taxonomía y Ecología de Peces, CINVESTAV-IPN, Unidad Mérida, km 6 antigua carretera a Progreso. \\ AP 73 Cordemex. 97310 Mérida, Yucatán; México; walterg11@yahoo.com.mx
}

\author{
Received 13-VIII-2007. Corrected 30-VI-2008. Accepted 31-VII-2008.
}

\begin{abstract}
Feeding habits of the fish Lagodon rhomboides (Perciformes: Sparidae) at the coastal lagoon of Chelem, Yucatán, México. Stomach contents of Lagodon rhomboides, the most abundant fish species from seagrass beds in Chelem Lagoon, Yucatan, Mexico, were analyzed. The specimens were collected using a beach seine at eight stations distributed randomly in the lagoon during July, September and November 2002. The trophic components were analyzed by means of the relative abundance $(\% \mathrm{~A})$ and frequency of occurrence (FO) indices. The trophic similarity between different ontogenetic stages was determined using the Bray-Curtis Index. A total of 90 stomach contents were analyzed. This species is omnivorous, including vegetal and animal material and has a wide trophic spectrum with 58 alimentary items. Trophic ontogenetic variation was significant with a transition from one feeding stage to the next. Small individuals (4.0 -8.0 cm LE) preferentially consume plankton preys and microcrustaceans, while in bigger sizes, the macrocrustaceans, annelids and macrophytes were the main food. Rev. Biol. Trop. 56 (4): 1837-1846. Epub 2008 December 12.
\end{abstract}

Key words: Trophic preferences, Lagodon rhomboides, stomach content analysis, Chelem lagoon, Mexico.

El estudio de los hábitos alimenticios y de la dieta en los peces nos lleva a comprender muchos aspectos de la biología, ecología, fisiología y fundamentalmente para un mejor entendimiento del rol funcional del necton en los diferentes ecosistemas acuáticos (Blaber 1997, Wootton 1998, Hajisamae et al. 2003). El análisis del contenido estomacal se ha convertido en el método más conocido y utilizado para el estudio de la dieta de los peces (Valente 1992). Éste tiende a ser usado para describir la trofodinámica en forma individual o de poblaciones, y así poder examinar la superposición del nicho ecológico y la competencia e interacciones intraespecífica e interespecíficas (Graham y Vrijenhoek 1988), además de determinar el rol de una especie en la cadena trófica de los ecosistemas costeros y marinos (Hyslop 1980, Valente 1992) y la función de los ecosistemas a través de modelos tróficos (Vega y Arreguín 2004). La dieta de los peces refleja la variabilidad de presas y por medio del contenido estomacal se puede tener una idea de la variabilidad de los ítems alimenticios en el medioambiente acuático en el cual se encuentran y/o habitan.

Lagodon rhomboides (Linnaeus), conocida localmente como xlavita, es una especie estuarino-dependiente cuyo ámbito de distribución incluye toda la costa de Massachussets, sur de Florida, Golfo de México y Península de Yucatán (Burguess 1980). Es común en las bahías, estuarios y lagunas costeras, sobre todo en praderas de Thalassia testudinum y en raíces de manglar (Adams et al. 2004). Usualmente se agrupan en cardúmenes y se caracteriza por ser un consumidor secundario (Vega-Cendejas et al. 1997). A finales de invierno o principios de primavera migran de las áreas costeras hacia zonas más profundas para su desove y en invierno los alevines retornan a los estuarios y lagunas costeras (Potthoff y Allen 2003; 
Shervette et al. 2007). Su importancia comercial radica en las pesquerías locales, ya que se comercializa entero o en filete entre la gente de la región (Vega-Cendejas et al. 1997).

La xlavita es una de las especies dominantes numéricamente de las aguas interiores del NE del Golfo de México (Livingston 1984; Stoner y Livingston 1984). Particularmente en la Península de Yucatán, ha sido reportada como una de las especies más representativas de las praderas de pastos marinos (Thalassia testudinum) en los sistemas lagunares de Celestún (Vega-Cendejas 1998), Chelem (Hernández 2002; Vargas 2004) y bocas de Dzilam (Arceo 2005). Específicamente en Chelem, representa el 53\% de la densidad del total de las especies capturadas (Vargas 2004). No obstante se han realizado estudios sobre la biología trófica de esta especie en los sistemas lagunares de Celestún (Vega-Cendejas et al. 1997, VegaCendejas 1998), se carece de un conocimiento del espectro alimenticio de L. rhomboides en el sistema lagunar de Chelem, así como de su variación con el crecimiento. Cabe resaltar que las preferencias alimenticias de una especie no solo dependen de los requerimientos energéticos específicos en las diversas etapas tróficas, sino también de las localidades y consecuentemente de la disponibilidad de la presa, por lo que el presente estudio es el primero de su índole en cuestión de profundidad y sobre todo en la laguna de Chelem, sistema que actualmente está sometido a un fuerte impacto turístico por su cercanía con el muelle de Progreso. En este sentido, el objetivo de este estudio es describir los hábitos alimenticios de L. rhomboides del sistema costero lagunar de Chelem, Yucatán, identificar los principales componentes que constituyen su alimentación y determinar las unidades tróficas ontogénicas.

\section{MATERIALES Y MÉTODOS}

Área de estudio: La laguna de Chelem se ubica al noreste del Estado de Yucatán, a $30 \mathrm{~km}$ al norte de la ciudad de Mérida $\left(21^{\circ} 15^{\prime}-21^{\circ} 17^{\prime} \mathrm{N}\right.$ y $\left.89^{\circ} 39^{\prime}-89^{\circ} 48^{\prime} \mathrm{W}\right)$. Presenta una superficie aproximada de $15 \mathrm{~km}$ y profundidad entre 0.5 y $1.5 \mathrm{~m}$. La temperatura media anual se encuentra entre los 24 y $26^{\circ} \mathrm{C}$. La vegetación que la rodea es manglar de bajo desarrollo principalmente Avicennia germinans y Rhizophora mangle, el fondo se encuentra cubierto principalmente por Thalassia testudinum (Herrera-Silveira el al. 1998).

Muestreo: Seis muestreos bimensuales se realizaron de marzo de 2002 a enero del 2003 en la laguna de Chelem, utilizando un chinchorro playero de $15 \mathrm{~m}$ de largo por $1.5 \mathrm{~m}$ de caída y apertura de malla de una pulgada, en ocho estaciones distribuidas aleatoriamente en el sistema. Todos los arrastres fueron efectuados entre las 07:00 y las 16:00 h. Los ejemplares recolectados se preservaron en formaldehído al $30 \%$ y fueron transportados al laboratorio para su identificación utilizando bibliografía especializada (Guitar 1974, Fischer 1978). Del total de ejemplares obtenidos, se registró individualmente el peso total (g) y la longitud estándar (LE) y se procedió a su análisis trófico. Cada entidad alimenticia se clasificó hasta el nivel taxonómico más bajo posible empleando un microscopio estereoscópico u óptico, según el grado de digestión de las presas, las cuales se clasificaron en grupos tróficos. Para estimar la variación trófica ontogénica se establecieron de forma arbitraria clases de longitud de $1.0 \mathrm{~cm}$, obteniendo en total 6 intervalos o clases de tallas.

El espectro alimenticio de L. rhomboides se describió a través del método de Porcentaje por Área (PA), el cual es una modificación del método volumétrico indirecto utilizado cuando el contenido estomacal está constituido por presas muy pequeñas (diatomeas, copépodos, ostrácodos) (Vega-Cendejas 1990). La composición de la dieta se expresó como porcentaje de su Frecuencia de Presencia (FO); FO = (n/ NE) (100), donde "n" se refiere al número de veces que aparece una determinada presa en el contenido estomacal y se expresa como el porcentaje con respecto al número total de estómagos analizados (N) (Tresierra y Culquichicóm 1993). Este método se utilizó como complemento del PA para precisar si el componente 
alimenticio es preferencial ( $\mathrm{FO}>50 \%$ ), secundario $(50 \%>\mathrm{FO}>10 \%)$ o incidental $(\mathrm{FO}<$ $10 \%$ ) cuando su consumo es ocasional.

La variación del espectro alimenticio con el incremento en talla, se identificó con un análisis de conglomerados en base a la composición y abundancia de las presas utilizando el índice de Bray-Curtis (Bray y Curtis 1957). Los datos fueron transformados a raíz cuarta para reducir la importancia de las presas más abundantes entre las tallas (Field et al. 1982). La contribución de cada presa en cada grupo de talla, se determinó a través de la prueba estadística SIMPER (porcentaje de similitud). Esta prueba determina el porcentaje de la contribución de cada presa a fin de clasificar un grupo (similaridad) y discriminar las presas entre los grupos (disimilaridad) (Clarke and Green 1988). Los análisis utilizados para determinar la variación trófica entre tallas y las preferencias alimenticias, fueron realizados con el programa estadístico PRIMER 5 (Clarke y Gorley 2001).

\section{RESULTADOS}

Biología trófica: Se obtuvieron un total de 90 ejemplares de Lagodon rhomboides con tallas entre 4.0 y $10.0 \mathrm{~cm}$ de LE. Del total de los estómagos analizados el $14.4 \%$ estaban llenos con material alimenticio identificable y el $85.6 \%$ se encontraban con algo de alimento. De acuerdo con el análisis del contenido estomacal, se observó que la dieta está constituida principalmente de material tanto de origen vegetal como animal. Es importante mencionar que en marzo (2002) y enero (2003) no se capturó ningún ejemplar de esta especie.

Composición de la dieta: A partir de los resultados se determinó que esta especie es un generalista trófico, incluyendo 58 componentes alimenticios. Los taxones identificados se distribuyeron en 14 grupos tróficos, dentro de los componentes alimenticios se registraron el fitoplancton, zooplancton, moluscos, macrófitas, microcrustáceos y macrocrustáceos, así como anélidos y peces (Cuadro 1).
Respecto a la abundancia (PA), el grupo de fitoplancton es el más representativo (30.94\%), siguiéndole en importancia los microcrustáceos (20.59\%), macrocrustáceos (17.84\%), macrófitas $(11.42 \%)$ y peces (5.99\%) (Cuadro 1$)$. Respecto a su presencia, sobresalen como presas preferenciales ( $\mathrm{FO}>50 \%$ ) los pastos dentro de grupo de las macrófitas, los copépodos y los ostrácodos. Las presas secundarias $(\mathrm{FO}<50 \%)$ fueron 28 , de los cuales sobresalen diversos géneros de diatomeas (Gyrosigma sp., Navicula spp., Fragilaria sp., Pinnularia sp., Melosira sp. y Amphora spp.), las semillas de algas, los macrocrustáceos de la familia Caridea, los microcrustáceos como los anfípodos y los isópodos (familia Cirolanidae), los foraminíferos del género Triloculina sp. y los dinoflagelados con el género Peridinium sp. Los componentes alimenticios con FO inferiores al 10\% se consideraron como incidentales (Cuadro 1).

Variación trófica ontogénica: Ejemplares de $L$. rhomboides con tallas entre 4.0 y $10.0 \mathrm{~cm}$ de LE, presentan una tendencia con el incremento de longitud, a disminuir el consumo de fito-zooplancton y microcrustaceos; por el contrario, el consumo de macrófitas, macrocrustáceos y peces se incrementa, contribuyendo con más del 54\% del contenido estomacal (Fig. 1). Considerando está variación trófica entre las tallas de los ejemplares de L. rhomboides analizados, se observa en la Figura 2 que a un corte de $15 \%$ de disimilitud, la presencia de tres etapas tróficas ontogénicas, las cuales se caracterizan por la abundancia de las presas preferenciales de cada unidad trófica (Fig. 1). En la primera etapa, ejemplares de tallas comprendidas entre 4.0-5.0 cm LE consumen preferentemente $83.81 \%$ del total de la dieta de fitoplancton y zooplancton, junto con $28 \%$ de microcrustaceos y $12 \%$ gasterópodos. La etapa II agrupa varios intervalos de talla, con una tendencia a la disminución de estos componentes, pero donde todavía el consumo de ellos es notable. Finalmente en la etapa III (9.1-10 cm LE), la ingestión de ambos componentes planctónicos decrece a $22.04 \%$, así como el de los microcrustáceos (3.8\%), mientras que el 
CUADRO 1

Composición del alimento de la xlavita (Lagodon rhomboides) en la laguna de Chelem, Yucatán, expresada como abundancia relativa (PA) y frecuencia porcentual de presencia $(F O)(N=90)$

\begin{tabular}{|c|c|c|c|c|c|}
\hline Artículo alimentario & PA & $\%$ FO & Artículo alimentario & PA & $\% \mathrm{FO}$ \\
\hline Fitoplancton & & & Porifera & & \\
\hline Bacillariophyceae & & & Esponjas & 0.02 & 1.11 \\
\hline Amphiprora sp. & 0.29 & 6.67 & Espículas de Esponjas & 0.52 & 16.67 \\
\hline Amphora spp. & 2.93 & 31.11 & Total & 0.54 & 17.78 \\
\hline Biddulphia sp. & 0.01 & 1.11 & Annelidae & & \\
\hline Cymatosira sp & 0.08 & 2.22 & Polychaeta & 3.92 & 22.22 \\
\hline Cocconeis sp & 0.06 & 2.22 & Total & 3.92 & 20.00 \\
\hline Coscinodiscus sp. & 0.02 & 2.22 & Mollusca & & \\
\hline Cyclotella sp. & 0.05 & 2.22 & Gasteropoda & & \\
\hline Diploneis sp & 0.09 & 3.33 & Crepidulidae & 3.57 & 17.78 \\
\hline Epithemia sp. & 0.40 & 5.56 & Marginellidae & 0.03 & 5.56 \\
\hline Eunotia sp. & 0.36 & 6.67 & Modillidae & 0.10 & 4.44 \\
\hline Fragilaria sp. & 3.68 & 38.89 & Total & 3.70 & 18.89 \\
\hline Grammatophora sp. & 1.04 & 27.78 & Microcrustáceos & & \\
\hline Gyrosigma sp. & 4.69 & 43.33 & Amphipoda & 6.84 & 26.67 \\
\hline Licmophora sp. & 0.02 & 1.11 & Copepoda & 3.29 & 56.67 \\
\hline Melosira sp. & 1.86 & 34.44 & Isopoda & & \\
\hline Navicula spp. & 4.48 & 40.00 & Sphaeromidae & 0.03 & 1.11 \\
\hline Neidium sp. & 0.02 & 1.11 & Cirolanidae & 3.87 & 25.56 \\
\hline Nitzschia spp. & 1.93 & 23.33 & Ostracoda & 5.60 & 55.56 \\
\hline Pinnularia sp. & 1.92 & 36.67 & Tanaidacea & 0.84 & 11.11 \\
\hline Podocystis sp. & 0.06 & 1.11 & Mysidacea & 0.12 & 1.11 \\
\hline Rhopalodia sp. & 0.01 & 1.11 & Total & 20.59 & 75.56 \\
\hline Striatella sp. & 1.82 & 28.89 & Macrocrustáceos & & \\
\hline Synedra sp. & 1.28 & 15.56 & Decapoda & & \\
\hline Total & 27.10 & 62.22 & Caridea & 16.37 & 30.00 \\
\hline Chlorophyceae & & & Penaeidae & 0.75 & 4.44 \\
\hline Cosmarium sp. & 0.02 & 1.11 & Cirripedia & & \\
\hline Desmidium sp. & 0.51 & 10.00 & Balanus sp. & 0.72 & 1.11 \\
\hline Total & 0.53 & 7.78 & Total & 17.84 & \\
\hline Cianophyceae & & & Peces & & \\
\hline Croococcus sp. & 0.02 & 1.11 & Peces no ident. & 5.99 & 22.22 \\
\hline Merismopedia sp. & 0.26 & 11.11 & Total & 5.99 & \\
\hline Microcystis sp. & 0.42 & 8.89 & Detritus & & \\
\hline Oscillatoria $\mathrm{sp}$. & 0.61 & 13.33 & Detritus & 2.26 & 14.44 \\
\hline
\end{tabular}


CUADRO 1 (Continuación)

Composición del alimento de la xlavita (Lagodon rhomboides) en la laguna de Chelem, Yucatán, expresada como abundancia relativa (PA) y frecuencia porcentual de presencia (FO) $(N=90)$

$\begin{array}{lrrlcc}\text { Artículo alimentario } & \text { PA } & \% \text { FO } & \text { Artículo alimentario } & \text { PA } & \% \text { FO } \\ \text { Total } & 1.31 & 15.56 & \begin{array}{l}\text { Total } \\ \text { Otros }\end{array} & 2.26 & \\ \text { Dinoflagellida } & & & & 0.21 & 6.67 \\ \text { Peridinium sp. } & 1.40 & 25.56 & \begin{array}{l}\text { Huevos no ident. } \\ \text { Bivalvia }\end{array} & 0.05 & 2.22 \\ \text { Total } & 1.40 & & \text { Nemátodo no ident. } & 0.11 & 3.33 \\ \text { Macrófitas } & & & 0.79 & 7.78 \\ \text { Pasto no ident. } & 7.91 & 65.56 & \text { Total } & & \\ \text { Algas no ident. } & 0.99 & 13.33 & & & \\ \text { Semillas de algas } & 2.52 & 35.56 & & \\ \text { Total } & 11.42 & 74.44 & & \\ \text { Protozoa } & & & & \\ \text { Foraminiferida } & & & & \\ \text { Globigerina } \text { sp. } & 0.23 & 7.78 & & \\ \text { Spiroculina } \text { sp. } & 0.74 & 12.22 & & \\ \text { Triloculina } \text { sp. } & 1.64 & 23.33 & & \\ \text { Total } & 2.61 & 16.67 & \end{array}$

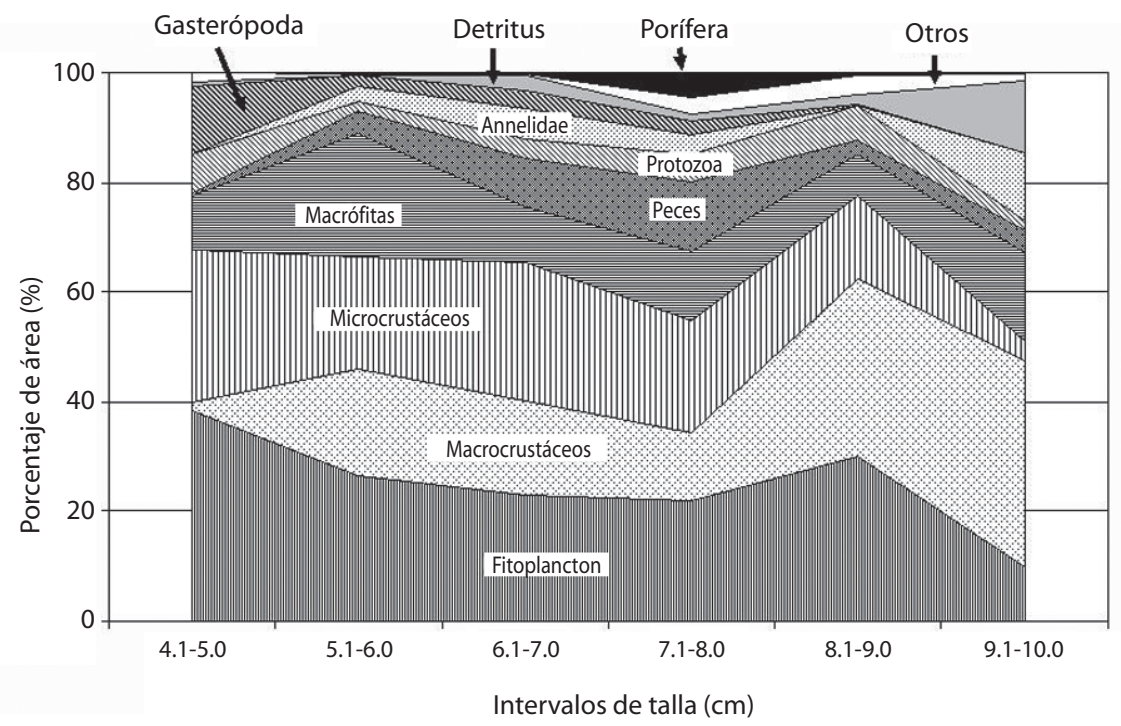

Fig. 1. Variación del espectro trófico (PA) entre diferentes intervalos de talla de Lagodon rhomboides, durante julio, setiembre y noviembre de 2002, en la laguna de Chelem Yucatán, México. 


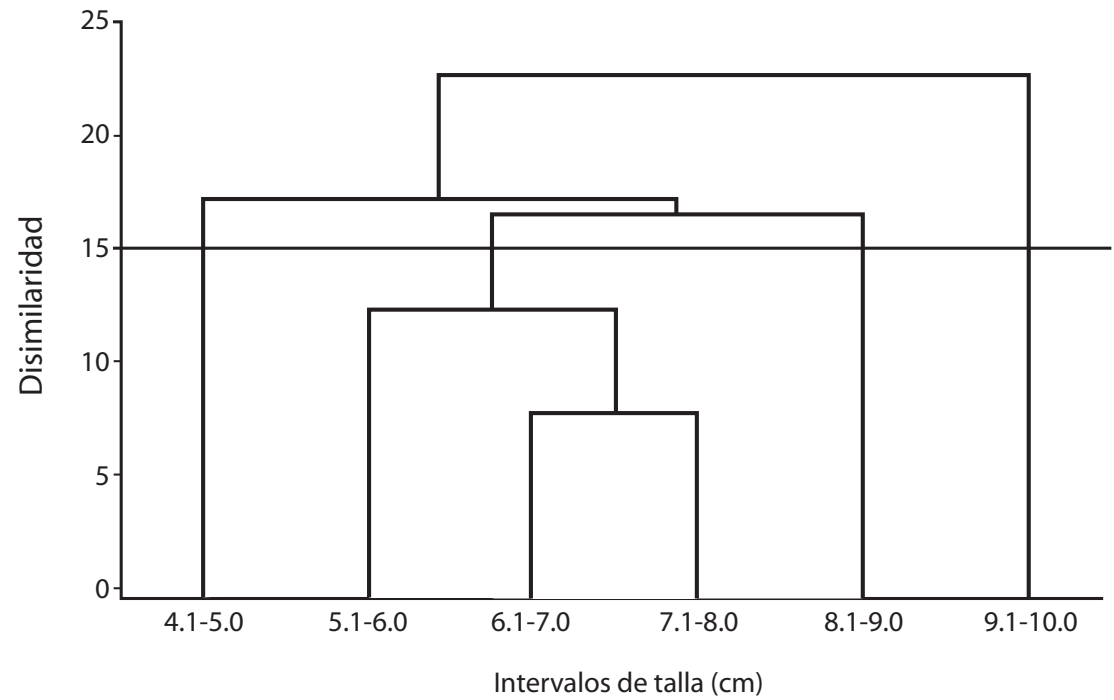

Fig. 2. Dendrograma de similitud trófica a partir del índice de Bray-Curtis (Bray y Curtis, 1957) que representa a las diferentes unidades tróficas durante la ontogenia de Lagodon rhomboides durante julio, setiembre y noviembre, en la laguna de Chelem Yucatán, México.

consumo de macrocrustáceos, principalmente carideos y pastos marinos se incrementa alcanzando valores de abundancia del 38 y $16 \%$, respectivamente del total de la dieta.

\section{DISCUSIÓN}

Los resultados de este estudio confirman que Lagodon rhomboides es una especie omnívora, consumiendo de manera significativa material vegetal y animal. Además de presentar una amplia generalización trófica al incluir en su dieta diversas presas en la laguna de Chelem. Esto se puede afirmar debido a la elevada cantidad de componentes alimenticios identificados en los estómagos (58) que incluyen el fitoplancton, microcrustáceos, macrófitas, macrocrustáceos y peces. Esta especie penetra a estos sistemas en etapas tempranas de desarrollo, utilizando las praderas deThalassia testudinum para protección y alimentación, ya que estos ambientes presenta una alta productividad primaria, la cual incrementa con las algas bénticas y epifitas, garantizando un aumento de materia orgánica que puede ser utilizada como fuente de energía básica para diversos organismos (microcrustáceos y peces) (Hemminga y Duarte 2000).

Respecto a la abundancia el grupo de fitoplancton fue el alimento más representativo, esto es debido a que en la laguna de Chelem durante los meses en los que se realizó el estudio los valores de productividad se incrementan por las altas temperaturas (28.3 ${ }^{\circ} \mathrm{C}$ ) y elevada salinidad (36.3) (Canto-Maza y Vega-Cendejas 2007), lo cual da lugar a procesos de descomposición de materia orgánica y mineralización, proporcionando un aporte significativo de nutrientes a la columna de agua que es aprovechado por el fitoplancton (Collí 2001,Vargas 2004). El segundo grupo en importancia fueron los microcrustáceos. Al respecto Potthoff y Allen (2003) mencionan 
que L. rhomboides juega un rol importante en los estuarios y lagunas costeras, como uno de los principales depredadores de invertebrados bentónicos. La dieta hallada durante este trabajo para L. rhomboides está en concordancia por lo reportada por Claro (1994) y Vega-Cendejas et al. (1998) en otros sistemas lagunares, los cuales la describen como una especie omnívora, teniendo como componentes principales en su dieta los crustáceos, moluscos, peces y vegetación. Por otra parte Rooker et al. (1998) encontró en un estuario en Texas a L. rhomboides como voraces depredadores, compitiendo por los recursos con otras especies estuarinodependientes, alimentándose de organismos similares como anfípodos y otros pequeños crustáceos como son los poliquetos y moluscos. Vega-Cendejas et al. (1997) la reportan en la laguna de Celestun como un consumidor de microcrustáceos bentónicos, incluyendo a los peces, material vegetal y poliquetos en menor cantidad.

Los ejemplares analizados de esta especie correspondieron a juveniles de 4.0 a $10.0 \mathrm{~cm}$ de LE (Potthoff y Allen 2003). Respecto a su alimentación, se observa una progresión de cambios en las preferencias alimenticias (Fig. 2). Los ejemplares de menor talla $(4.0-5.0 \mathrm{~cm}$ LE) son prácticamente planctívoros, alimentándose principalmente de fitoplancton como Fragilaria y Navicula, que son consumidas con una presencia del 39 y $40 \%$, respectivamente. Con el crecimiento hay una transición gradual hacia la carnivoría béntica en la forma de anfípodos, copépodos harcapticoideos, ostrócodos y otros pequeños invertebrados. Ejemplares $>9.0 \mathrm{~cm} \mathrm{LE}$, incrementan la preferencia por macrocustaceos, donde los carideos, macrófita, anélidos y detritus son las presas de mayor abundancia y presencia (Cuadro 1).

Estos resultan coinciden con los resportados por Livingston (2003) en Bahía Apalache donde ejemplares de L. rhomboides $>12 \mathrm{~cm}$ de LE, se alimentan generalmente de materia vegetal en la forma de Syringodium y Thalassia. Vega-Cendejas (1998) en la laguna de Celestún, reporta que ejemplares con tallas entre 3.0 a 7.0 de LE consumen principalmente fitoplancton y microcrustáceos, mientras que con el crecimiento incorporan a la materia vegetal. In Isla Contoy, los peces con tallas < $7.0 \mathrm{~cm}$ LS incluyen además de estos componentes alimenticios al detritus y esponjas (VegaCendejas et al. 2000). Por otra parte, también ha sido descrita por otros autores como una especie omnívora y con una alta generalización trófica al incluir en su alimentación diversos grupos tróficos en función de sus necesidades y de la disponibilidad en el medio (Hansen 1969, Adams 1976, Stoner 1980, Weinstein et al. 1982, Vega-Cendejas y Hernández 1991, VegaCendejas et al. 1994). Potthoff y Allen (2003) mencionan que $L$. rhomboides es reconocidos por su voracidad y agresividad en su forma de alimentarse, además de que compiten con otras especies del necton por el espacio y el recurso.

Las transiciones de una etapa a otra son a menudo graduales y no necesarias distintas, pero están generalmente relacionadas a las etapas de crecimiento en el desarrollo ontogénico de cada especie (Livingston 2003). Stoner y Livingston (1984) resaltan que las progresiones alimenticias están asociadas con cambios ontologicos respecto a la morfología y correlacionados con el movimiento (forma del cuerpo), dentición y dimensiones de la boca e intestino. Al respecto, podemos mencionar que el patrón de alimentación basada en fitoplancton y microcrustáceos (fuente rica en proteínas) en ejemplares de menor talla podría estar en función de los requerimientos energéticos para su crecimiento (Deudero 2001). Por otro lado, el consumo de microcrustáceos en las etapas tempranas del desarrollo puede deberse también a su disponibilidad en el medio (Poot-Salazar et al. 2005). Otro aspecto importante de estos cambios ontogénicos en la dieta, es que permiten una repartición de los recursos con otras especies, generando de esta forma mayores tasas de crecimiento entre ellas y reduciendo la competencia interespecífica (García-Berthou y Moreno-Amich 2000). Esto puede estar sucediendo, ya que resultados de trabajos anteriores de los hábitos alimenticios de Floridichthys polyommus y Opsanus phobetron en la laguna de Chelem, mostraron 
que también son consumidores de microcrustáceos, fitoplancton y macrófitas además de otros grupos tróficos (Canto-Maza 2004, PootSalazar et al. 2005).

\section{AGRADECIMIENTOS}

Agradecemos a Mirella Hernández de Santillana por la ayuda proporcionada en la recolecta de muestras y en la identificación de los contenidos estomacales, así como al grupo de trabajo que apoyó en las actividades de campo y laboratorio. Por otra parte deseamos externar nuestro agradecimiento a los revisores anónimos cuyos comentarios y sugerencias enriquecieron el presente documento.

\section{RESUMEN}

Se analizó el contenido estomacal de Lagodon rhomboides (Sparidae), la especie más abundante de pez en la zona de pastos marinos en la laguna de Chelem, Yucatán, México. Los especímenes se recolectaron utilizando un chinchorro playero en ocho estaciones distribuidas aleatoriamente durante julio, septiembre y noviembre del 2002. Los componentes tróficos fueron analizados por medio del porcentaje de la abundancia relativa $(\% \mathrm{~A})$ y la frecuencia de presencia (FO). La similitud trófica entre las diferentes etapas ontogénicas fue determinada usando el índice de Bray-Curtis. Se analizaron un total de 90 contenidos estomacales. Esta especie es omnívora e incluye en su alimentación componentes tanto de origen vegetal como animal; muestra una amplia generalización trófica con 58 componentes alimenticios. La variación trófica ontogénica fue significativa con una progresión alimenticia de una etapa alimenticia a la siguiente. Los individuos de menos talla, consumen preferentemente presas planctónicas y microcrustáceos s (4.0 - $8.0 \mathrm{~cm}$ de LE), mientras que en los de mayor talla, los macrocrustáceos (carideos), poliquetos y macrófitas constituyen el alimento principal.

Palabras clave: Preferencia trófica, Lagodon rhomboides, análisis del contenido estomacal, Laguna de Chelem, México.

\section{REFERENCIAS}

Adams, M.S. 1976. The Ecology of eelgrass, Zostera mari$n a$ (L.), fish communities. II. Functional Analysis. J. Exp. Mar. Biol. Ecol. 22: 193-311.

Adams, A. J., J.V. Locasciob \& B.D. Robbins. 2004. Microhabitat use by a post-settlement stage estuarine fish: evidence from relative abundance and predation among habitats. J. Exp. Mar. Biol Ecol. 299: 17- 33.

Arceo C., D. 2005. Ensamblajes de los peces de la Reserva Bocas de Dzilam, Yucatán y su relación con variables hidrológicas. Maestría en Ciencias, especialidad en Biología Marina. CINVESTAV - Unidad Mérida. $74 \mathrm{p}$.

Blaber, J.M. 1997. Fishand and fisheries of tropical estuaries. Capman y Hall.

Bray, J. R. \& J. T. Curtis. 1957. An ordination of the upland forest communities of Southern Winsconsin. Ecological Monographs 27:235-249.

Burgess, G.H. 1980. Pinfish. In: Lee DS (ed) Atlas of North American freshwater fishes. Publication No. 1980-12 N.C. Biological Survey. Carolina del Norte, EEUU.

Canto-Maza, W.G. 2004. Análisis trófico de las especies ícticas dominantes en el estero de Yucalpetén. Tesis de Licenciatura, Instituto Tecnológico Agropecuario, Conkal, Yucatán, México.

Canto -Maza, W.G. \& M.E. Vega-Cendejas. 2007. Distribución, abundancia y preferencias alimenticias del pez sapo Opsanus phobetron (Batrachoididae) en la laguna costera de Chelem Yucatán, México. Rev. Biol. Trop. 55: 979-988.

Clarke KR, Green RH (1988) Statistical design and analysis for a 'biological effects' study, Marine Ecology Progress Series 46: 213-226

Clarke K. R. \& R. Gorley 2001. PRIMER V5: User Manual/Tutorial. PRIMER-E, PLYMOUTH, Reino Unido.

Claro, R. 1994. Ecología de los peces marinos de cuba. Centro de Investigación de Quintana Roo. Academia. Quintana Roo, México.

Collí, R.C. 2001. Diagnóstico de la salud ambiental de la Laguna de Chelem. Tesis de Maestría. Instituto Tecnológico de Mérida. Yucatán, México.

Deudero, S. 2001. Interspecific trophic relationship among pelagic fish species underneath FADs. J. Fish Biol. 58: 53-67.

Field, J. G., K. R. Clarke \& R. M. Warwick. 1982. A practical strategy for analysis multispecies distribution patterns. Marine Ecology Progress Series 8:37-52.

Fischer, W. 1978. FAO Species Identification Sheets for Fishery Purposes. Western central Atlantic. FAO, Roma, Italia. 
García-Berthou, E. \& R. Moreno-Amich. 2000. Food of introduced pumpkinseed sunfish: ontogenetic diet shift and seasonal variation. J. Fish Biol. 57: 29-40.

Graham, J.H. \& R.C. Vrijenhoek. 1988. Detrended correspondence analysis of dietary data. Transactions of the American Fisheries Society. 117:29-36.

Guitar, D.J. 1974. Sinopsis de los Peces Marinos de Cuba. Academia de Ciencias de Cuba. Instituto de Oceanología. La Habana, Cuba.

Hajisamae, S., L.M. Chou \& S. Ibrahim. 2003. Feeding habits and trophic organization of the fish community in hallow waters of an impacted tropical habitat. Estuar. Coast. Shelf Sci. 58:89-98.

Hansen, D.J. 1969. Food, growth, migratión, reproductión and abundance of pinfish, Lagodon rhomboides, and Atlantic croaker, Micropogonias undulatus, near Pensacola, Florida. Fish. Bull. 68: 135-146.

Hemminga, M.A. \& C.M. Duarte. 2000. Seagrass Ecology. Cambridge University. United Kingdom.

Hernández V., T. P. 2002. Estructura de la comunidad y ensamblaje de peces juveniles, en la laguna de Chelem, Yucatán (Nortes-Secas). Benemérita Univ. Autónoma de Puebla. Escuela de Biología. México Distrito Federal, México.

Herrera-Silveira, J.A., R.J. Ramírez, \& J.A. Zaldivar. 1998. Overview and characterization of the hydrology and primary producer communities of selected coastal lagoons of Yucatan, México. Aqua. Ecosyst. Health Manag. 1: 353-372

Horn, H.S. 1966. Measurement of overlap in comparative ecological studies. Am. Nat. 100: 419-424.

Hyslop, E.J. 1980. Stomach contents analysis: a review of methods and their application. J. Fish Biol. 17: 411-429.

Livingston, R.J. 1984. Trophic response of fishes to habitat variability in coastal seagrass systems. Ecology 65 : 1258-1275.

Livingston, R.J. 2003. Trophic organization in coastal systems. CRC. Florida University, Tallahassee, Florida, EEUU.

Poot-Salazar, A. V., W. G. Canto-Maza \& M. E. VegaCendejas. 2005. Hábitos alimenticios de Floridichthys polyommus Hubbs, 1936 (Pisces: Cyprinodontidae) en dos sistemas lagunares costeros. Hidrobiológica. 15: 183-194.
Potthoff, M.T. \& D.M. Allen. 2003. Site fidelity, home range, and tidal migrations of juvenile pinfish, Lagodon rhomboides, in salt marsh creeks. Environ. Biol. Fish. 67:231-240.

Rooker. J.R., G.J. Holt \& S.A. Holt. 1998. Vulnerability of newly settled red drum, Sciaenops ocellatus, to predatory fish: is early life survival enhanced by seagrass meadows. Mar. Biol. 131:145-151.

Shervette, V. R., N. Ibarra \& F. Gelwick. 2007. Influences of salinity on growth and survival of juvenile pinfish Lagodon rhomboides (Linnaeus). Environ. Biol. Fish (2007) 78:125-134.

Stoner, W.A. 1980. Feeding ecology of Lagodon rhomboides (pisces: Sparidae): Variation and functional responses. Fish. Bull. 78: 337-352.

Stoner, W.A. \& R. J. Livingston, 1984. Ontogenetic patterns in diet and feeding morphology in sympatric sparid fishes from seagrass meadows. Copeia (1984): 174-187.

Tresierra, A.A. \& M.Z. Culquichicóm. 1993. Biología pesquera. Libertad. Trujillo, México Distrito Federal, México.

Valente A., C.N. 1992. A alimentacao natural dos peixes (métodos de estudo). Instituto de Zoología Dr Augusto Nobre, Universidade do Porto. Série Monografias, 4.

Vargas, K.E. 2004. Composición y Distribución íctica, durante la temporada de lluvias y nortes en el estero de Yucalpetén, Yucatán, México. Tesis de Licenciatura. Universidad Autónoma de Yucatán, México.

Vega-Cendejas, M.E. 1990. Interacción trófica entre dos bagres Arius melanopus (Agassiz, 1829) y Arius felis (Linnaeus, 1776) en las costas de Celestún Yucatán, México. An. Inst. Cienc. Del Mar y Limnol. Univ. Nal. Auton. México. 15: 185-194.

Vega-Cendejas, M.E. 1998. Trama trófica de la comunidad nectónica asociada al ecosistema de manglar en el litoral Norte de Yucatán. Tesis de Doctorado. Fac. De Ciencias. Univ. Nal. Autón. México. Distrito Federal, México.

Vega-Cendejas, M.E. \& M.J. Hernández de S. 1991. Aspecto trófico de la Xlavita, Lagodon rhomboides de las costas de Celestún Yucatán, México. XI Congreso de Zoología, México DF.

Vega-Cendejas, M. E. \& Arreguín- Sánchez, F. 2001. Energy fluxes in a mangrove ecosystem from a 
coastal lagoon in Yucatan Peninsula, Mexico. Ecol. Model. 137: 119-133.

Vega-Cendejas, M.E., M.J. Hernández de S. \& F. ArreguínSánchez. 1994. Trophic interrelationships of fishes in a beach seine fishery from the northwestern coast of the Yucatan, México. J. Fish Biol. 44: 647-659.

Vega-Cendejas, M.E., M.J. Hernández de S. \& A.G. Cruz. 1997. Los peces de la reserva de Celestún. Pronatura Península de Yucatán, A. C. The Nature Conservancy. CINVESTAV-Unidad Mérida. Yucatán, México.

Vega-Cendejas, M.E., U. Ordóñez-López, M.J. Hernández de S., F. Mérino \& J. Hirose. 1998. Los peces de la reserva de Río Lagartos: complejo faunístico de gran valor ecológico, acuacultural y pesquero. Informe técnico final de proyecto de investigación. SISIERRA.
Recursos del Mar. Cinvestav-IPN, Unidad Mérida, Yucatán, México.

Vega-Cendejas, M.E., M.J. Hernández de S., G. Mexicano, G. Avilés, E. F. Vázquez \& G. V. Castillo. 2000. Informe técnico final del proyecto de investigación "Ecología y Evaluación de las Poblaciones de Peces en los Sistemas Costeros del Parque Marino Isla Contoy. Recursos del Mar. Cinvestav-IPN, Unidad Mérida, Yucatán, México.

Weinstein, M.P., K.L. Heck, P.F. Grebel \& J.E. Gates. 1982. The role of herbivory in pinfish (Lagodon rhomboides): a preliminary investigation. Bull. Mar. Sci. 32: 791-795.

Wootton, R.J. 1998. Ecology of Teleost Fishes. $2^{\mathrm{a}}$ ed. Chapman \& Hall. Londres, Inglaterra. 